\title{
Analog to Digital Conversion in Physical Measurements
}

\author{
T. Kapitaniak ${ }^{(1,2)}$, K. Życzkowski ${ }^{(1,3)}$, U. Feudel ${ }^{(1,4)}$ and C. Grebogi ${ }^{(1)}$ \\ ${ }^{(1)}$ Institute for Plasma Research, University of Maryland, College Park, MD 20742, USA \\ (2) Katedra Dynamiki, Politechnika Łódzka, ul. Stefanowskiego 1/15, 90-924 Łódź, Poland \\ (3) Instytut Fizyki im. Smoluchowskiego, Uniwersytet Jagielloński,ul. Reymonta 4, 30-059 Kraków, Poland \\ (4) Institut für Physik, Universität Potsdam, PF 601553, 14415 Potsdam, Germany \\ There exist measuring devices where an analog input is converted into a digital output. Such \\ converters can have a nonlinear internal dynamics. We show how measurements with such convert- \\ ing devices can be understood using concepts from symbolic dynamics. Our approach is based on a \\ nonlinear one-to-one mapping between the analog input and the digital output of the device. We \\ analyze the Bernoulli shift and the tent map which are realized in specific analog/digital converters. \\ Furthermore, we discuss the sources of errors that are inevitable in physical realizations of such \\ systems and suggest methods for error reduction. \\ PACS no. : 05.45. + b.
}

Progress in physics is based on the intimate relationship between experiment and theory. Experiments involve measurements in which the precision of the measured quantities depends essentially on the particular characteristics of the measuring devices. Often this process of measurement involves a conversion of the analog measured quantity into a digital number by means of a converter. For such analog/digital converters, there exists an internal relationship between the quantity to be measured (analog input), and the result of the measurement process (digital output). We assume that the conversion of the analog signal into a digital number is the sole process in such converters 
and we neglect errors associated with the input analog signal itself. Thus, the dynamics of the input-output relationship determines the whole conversion process and it is responsible for the precision of the conversion itself. This problem is especially relevant for converters based on nonlinear processes, for which it is known that the errors grow exponentially fast. In this paper, we analyze the properties of a such relationship in order to identify and understand the sources of possible errors involved in the conversion process.

As a paradigm of a device having a nonlinear internal dynamics, we consider the algorithmic analog/digital (A/D) converters [1]. The internal dynamics of such converters is described by well-known one-dimensional chaotic maps [2], the Bernoulli shift

$$
x_{n+1}=G_{1}\left(x_{n}\right)=2 x_{n} \quad \bmod 1
$$

and the tent map

$$
x_{n+1}=G_{2}\left(x_{n}\right)=1-2\left|x_{n}-\frac{1}{2}\right| .
$$

Both maps are characterized by a positive Lyapunov exponent $\lambda=\ln 2$ and they often serve as model examples in mathematical work devoted to the theory of dynamical systems (see e.g. [3]).

The Bernoulli shift is realized by the circuit shown in Fig. 1(a) 2]. This circuit represents a $K$-bit algorithmic analog/digital (A/D) converter, which is a recycling multistage converter with one bit stage. The converter achieves $K$-bit resolution by converting the input once and then circulating the residue function $G_{1}($.$) through the stage (K-1)$ times. The tent map is realized as a Grey-code algorithmic A/D converter [2] with a folding circuit having a transfer characteristic given by Eq. (2) as shown in Fig. 1(b).

To analyze the input-output relationship of such analog/digital converters from the point of view of the theory of dynamical systems, we apply concepts from symbolic dynamics. In analyzing the conversion process, we use the sensitive dependence on the initial conditions, a fundamental property of Eqn.(11) and (2), to argue that precise analog/digital conversion is possible. We identify the sources of errors in such converters and present a simple method of error reduction. 
Suppose we need to measure the quantity $X$, and let $\mathcal{X}$ denote the set of all possible values of $X$. Let us consider an unknown value $x_{1}$ of $X$, which is the analog input of the converter, as an initial condition that is iterated internally using the chaotic map $G$ generating the sequence $\left\{x_{k}\right\}=x_{1} x_{2} \ldots x_{L}$ with $x_{k}=G^{k-1}\left(x_{1}\right)$ and $k=1,2, \ldots, L$. To describe such an orbit in terms of a symbolic sequence, we need to choose a partition by coarse-graining the space $\mathcal{X}$. In the case corresponding to the maps (1) and (2), we split the space $\mathcal{X}=[0,1]$ into two cells whose partition is the critical point $x_{c}=1 / 2$. With any given orbit $\left\{x_{k}\right\}$ one can associate its symbol sequence $\left\{a_{k}\right\}$ using a two-letter alphabet defined as $a_{k}=0$ for $x_{k} \leq x_{c}$ and $a_{k}=1$ for $x_{k}>x_{c}$. The sequence $\left\{a_{k}\right\}$ is called the itinerary of $x_{1}$. Let $\mathcal{O}$ be a set of all possible symbolic sequences.

The process of conversion is pregnant with meaning if there exists a one-to-one mapping between $\mathcal{X}$ and $\mathcal{O}$. Once the itinerary characterizing the orbit is obtained, we get a result in form of a digital output

$$
y=\gamma\left(\left\{a_{k}\right\}\right)
$$

reflecting the unknown value $x_{1}$. In general, the exact form of the function $\gamma$ depends on the internal dynamics of the converter. For a dynamics given by the maps (1) and (2), it takes a simple form in a binary basis

$$
\gamma\left(\left\{a_{k}\right\}\right)=0 . c_{1} c_{2} \ldots c_{L}=\sum_{k=1}^{L} c_{k} 2^{-k}
$$

where

$$
c_{k}=a_{k},
$$

for the Bernoulli shift and

$$
c_{k}=\sum_{i=1}^{k} a_{i} \mid \bmod 2
$$

for the tent map [4]. The latter case is sometimes referred to as the Gray code [2].

The precision of such an analog/digital converter depends on the length $L$ of the itinerary. In principle, the digital output becomes arbitrarily precise for $L$ large enough, adding one binary digit at each internal iteration of Eqs. (11) or (2), as symbolized in Eq. (荬). In practice, the precision 
of the digital output is limited by several factors. The following main sources of errors in the conversion process may be identified:

(i) Inaccuracy in modeling: the internal dynamics of the converter results in a linear map with slope $s \neq 2$.

(ii) Partition point placed at $x_{c} \neq 1 / 2$.

These errors have a significant influence on the precision of the digital output. Errors of the above types destroy the one-to-one mapping between $\mathcal{X}$ and $\mathcal{O}$ as expressed by Eqs. (11) and (2). In order to deal with errors (i) and (ii), we address the following question: Can we still use converting devices with internal chaotic dynamics since errors grow exponentially fast in them?

To visualize the effect of changes in the slope (i), we present in Fig. 2 the plots of the inputoutput relations $y=f\left(x_{1}\right)$ for the Bernoulli shift [Fig. 2(a)] and for the tent map [Fig. 2(b)]. We choose the discrepancies in the slope $\left(\varepsilon_{s}=2-s\right)$ much larger than in practical applications, to demonstrate clearly the devil's staircase like character of this function. Let us denote by $\zeta$ the error of a single conversion, $\zeta=\left|y-x_{1}\right|$. The maximal error, $\Delta_{s}=\max _{x \in(0,1)} \zeta$, as well as the mean error, $\int_{0}^{1} \zeta(x) d x$, grow linearly with the discrepancy $\varepsilon_{s}$.

For our purpose, it is more important the error associated with a non-optimal placing of the partition point (ii) and how it influences the precision of the output. Figure 3 presents the inputoutput relationship $y\left(x_{1}\right)$ for the Bernoulli map (a) and the tent map (b) with the correct slope $(s=2)$ and a misplaced partition point $x_{c}=\frac{1}{2}-2^{-M}$ with $M=4$. The data are obtained using $L=24$ bits at each conversion. The comparison of these pictures clearly demonstrates the superiority of the tent map over the Bernoulli shift for conversion purposes. The Bernoulli shift is not continuous at $x_{c}=1 / 2$, which leads to large errors when the partition point is displaced.

To characterize the effect of misplacing the partition point quantitatively, we set an arbitrary tolerance level $\delta<<1$ and compute the cumulative probability $F(\delta):=\int_{\delta}^{1} P(\zeta) d \zeta$, the probability that the error exceeds a given tolerance level. The dependence $F(\delta)$ obtained for both maps 
is shown in Fig. 4 for a realistically possible misplacement $(M=8)$ of the partition point at $x_{c}=1 / 2-2^{-8} \approx 0.496$. Filled circles correspond to the tent map, which is less vulnerable to imperfections than the Bernoulli shift (empty circles).

To obtain analytical estimates of the errors, let us assume that the dependence shown in Fig. 3 is piecewise linear. If $x \in D=\left[x_{c}, 1 / 2\right]$ the error occurring for the Bernoulli shift is close to $1 / 2$. For any $x$ belonging to the first preimage of this interval, the error is approximately equal to $1 / 4$. Furthermore, for $x$ in the $n$-th preimage of the interval $D$, the error reads $2^{-1-n}$. The probability distribution of the error consists thus of the sum of singular Dirac deltas $P(\zeta)=$ $2^{-M}[\delta(\zeta-1 / 2)+\delta(\zeta-1 / 4)+\delta(\zeta-1 / 8)+\ldots]$. Calculating the cummulative distribution of error $F(\delta)$, we get $F\left(2^{-n}\right)=(n-1) 2^{-M}, n \geq 1$. In the variables used in Fig. 4, this relation reads $\log _{2} F\left(\log _{2} \delta\right)=-M+\log _{2}\left[-\log _{2} \delta-1\right]$.

A similar reasoning is applicable to the tent map. For any initial condition $x$ in the interval of the displacement $D=\left[x_{c}, 1 / 2\right]$, the error equals $2(1 / 2-x)$. In this approximation the distribution of error $P(\zeta)$ consists of a rectangle $P_{0}(\zeta)=1 / 2$ for $\zeta \in\left[0,2^{-M+1}\right]$. There exists two preimages of $D$, for which the errors belong to $\left[0,2^{-M}\right]$. Their contribution to the distribution of error reads $P_{1}(\zeta)=1$ for $\zeta \in\left[0,2^{-M}\right]$. In an analogous way, the errors generated by the $n$-th preimages of $D$ are described by $P_{n}(\zeta)=2^{n-1}$ for $\zeta \in\left[0,2^{-M+1-n}\right]$. To obtain the cumulative error distribution $F(\delta)$ we have to sum all terms arising from $P_{n}(\zeta), n=0,1,2, \ldots$ Making use of the sum of a geometric series we arrive at $F\left(2^{-M+1-n}\right)=\left[2 n-2+2^{1-n}\right] 2^{-M-1}$. Equivalently, a suitable expression for comparison with the numerical data reads $\log _{2} F\left(\log _{2} \delta\right)=-M-1+\log _{2}\left[-2\left(M+\log _{2} \delta\right)+2^{M+\log _{2} \delta}\right]$. Above error estimates, represented in Fig. 4 by continuous and dashed lines, respectively, describe the numerical data very well for both maps.

Since the errors caused by the misplacement of the partition point are mostly relevant for $x$ at any rational numbers of the type $k / 2^{n}$, with $n=1,2, \ldots$ and $k=1,2, \ldots, 2^{n}-1$, we propose a simple correction scheme based on the idea of a translation of the input value on the circle (or, equivalently, of the acting map). Consider a vector $\bar{v}=\left\{v_{1}, v_{2}, \ldots v_{N}\right\}$, in which $v_{1}=0$ and all other $N-1$ 
components are relatively incommensurate numbers from $(0,1)$. The conversion algorithm with a correction scheme consists in shifting the unknown quantity and obtaining $x_{1}^{(i)}=x_{1}+\left.v_{i}\right|_{\bmod 1}$, converting them by the procedure described above and obtaining $N$ different raw digital results $y^{(i)}$, and performing the reverse shift $\tilde{y}^{(i)}=y^{(i)}-\left.v_{i}\right|_{\bmod 1}$ with $i=1, \ldots, N$. Some of the results $\tilde{y}^{(i)}$, may be correct, some can be significantly wrong. Taking the average of these numbers would be rather meaningless; we propose to take for $N$ an odd number, order all the results $\tilde{y}^{(i)}$ and accept the middle one as a final result $y$. To test the practical relevance of this algorithm, we took $\bar{v}=\{0, \sqrt{2} / 2, \sqrt{3} / 3, \sqrt{5} / 5, \sqrt{7} / 7\}$ and use it for both maps discussed above. As shown in Fig. 4 $N=3$ (triangles) already reduces the total error by a factor close to ten. Taking the middle value of $N=5$ as the resulting output values significantly improves even the worse converter based on the Bernoulli shift (diamonds). The results for the continuous tent map with an $N=5$ correction are so good that the cumulative error, smaller than $2^{-20}$, is not marked in the picture.

The major conclusion of this work is that analog/digital converters with chaotic internal dynamics can be used for precise conversion of physical signals into digital numbers. We analyzed the sources of errors stemming from imperfections of the converter and propose an error correction algorithm capable of reducing the error of the conversion. In practice, the final precision of the digital output is governed by the accuracy of the auxiliary vector $\bar{v}$ but, nevertheless, an improvement gained by this procedure can be expected. Besides the errors discussed in this paper there are other possible errors leading to imprecisions of the digital output. Firstly, the internal dynamics of the device is nonlinear instead of piecewise linear. Secondly, the partition point location is fuzzy within a finite width $\epsilon>0$. The latter problem has been recently discussed in the context of communication with chaos [5].

We summarize the process of analog/digital conversion with internal nonlinear dynamics as given by the following steps:

1. Input of the analog signal $x_{1}$ to be measured into a nonlinear system $x_{n+1}=G\left(x_{n}\right)$ as an 
initial condition.

2. Generation of symbolic sequence $\left\{a_{k}\right\}$ by iterating the nonlinear map $\mathrm{G}($.$) .$

3. Calculation of $\gamma\left(\left\{a_{k}\right\}\right)$ according to formula (估).

4. Obtaining the digital output value $y$ given by Eq. (3) as the result of the measurement. 
We thank M. Ogorzałek and P. M. Kennedy for pointing us valuable references. T. K., K. Ż. and U. F. thank the University of Maryland for their hospitality. T. K. and K. Ż. acknowledge the Fulbright Fellowships while U. F. acknowledges the support from the Deutsche Forschungsgemeinschaft and from the Sonderforschungsbereich 555. C. G. was supported by DOE and by a joint NSF/CNPq grant.

\section{References}

[1] U. Fiedler and D. Seitzer, IEEE J. Solid-State Circuits SC-14, 547 (1979); P. W. Li, M. J. Chin, P. R. Gray and R. Castello, IEEE J. Solid-State Circuits SC-19, 828 (1984); C. S. G. Conroy, A High-Speed Parallel Pipeline A/D Converter Technique in CMOS, PhD-thesis, University of California at Berkeley; P. E. Pace, P. A.Ramamoorthy and D. Styer, IEEE Trans. Circuits Syst.II 41, 373 (1994); G. W. Roberts, Circuits and Systems Tutorials, IEEE ISCAS'94, London, 534 (1994).

[2] M. P. Kennedy, Int. J. Bifurc. \& Chaos, 5, 891 (1995).

[3] D. Gulick, Encounters with Chaos, McGrawHill, New York 1992.

[4] H. Bai-Lin, Elementary Symbolic Dynamics (World Scientific: Singapore, 1989)

[5] E. Bollt, Y.-C. Lai, and C. Grebogi, Phys. Rev. Lett. 79, 3787 (1997); J. Jacobs, E. Ott and B. R. Hunt, Phys. Rev. E 57, 6577 (1998); E. Bollt, Y.-C. Lai, Phys. Rev. E 58, 1724 (1998);

K. Życzkowski and E. Bollt, preprint chao-dyn/9807013. 


\section{CAPTIONS}

Figure 1: The circuit implementation of the Bernoulli shift (a) and the tent map (b).

Figure 2: Digital output $y$ as a function of the measured input value $x_{1}$. The conversion scheme is based on the dynamics of the Bernoulli shift (a) and the tent map (b) with the slopes $s=2.0$ (ideal case, diagonal) and non ideal cases $s=1.7$ and $s=1.4$ (thick line).

Figure 3: As in Fig. 2, the conversions have been performed with systems based on the Bernouli shift (a) and the tent map (b). Systematic errors are caused by the misplaced partition line localized at $x_{c}=\frac{1}{2}-2^{-4}$.

Figure 4: Cumulative probability $F$ of error larger than the tolerance level $\delta$ obtained for the Bernoulli shift (open symbols) and the tent map (full symbols) with the misplacement of the partition line characterized by $M=8$. The circles represent results received by measuring $L=24$ bits. Triangles and diamonds represent data obtained with $L=24$ by applying the $N=3$ and $N=5$ error-correction scheme. 
(a)

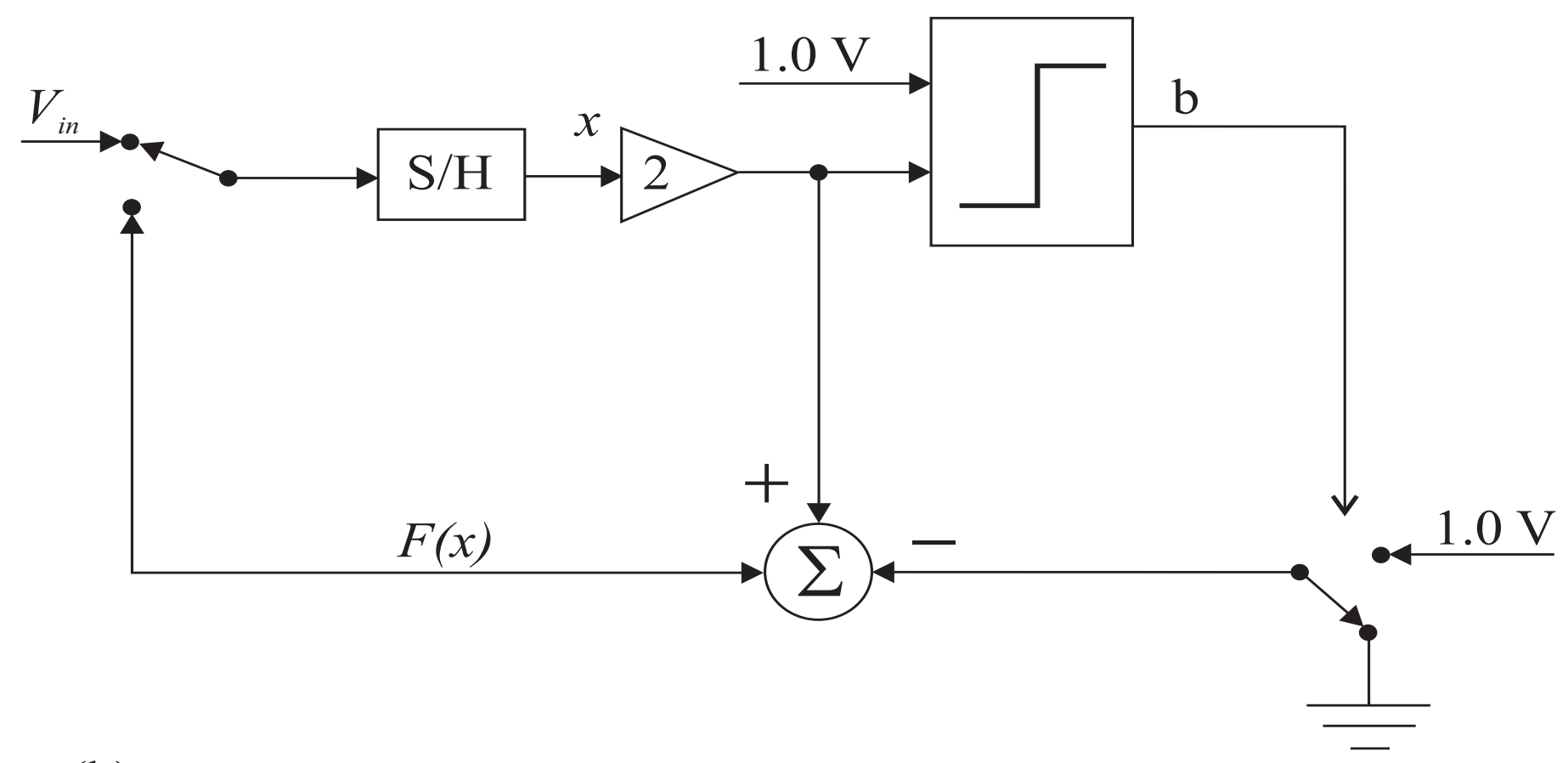

(b)

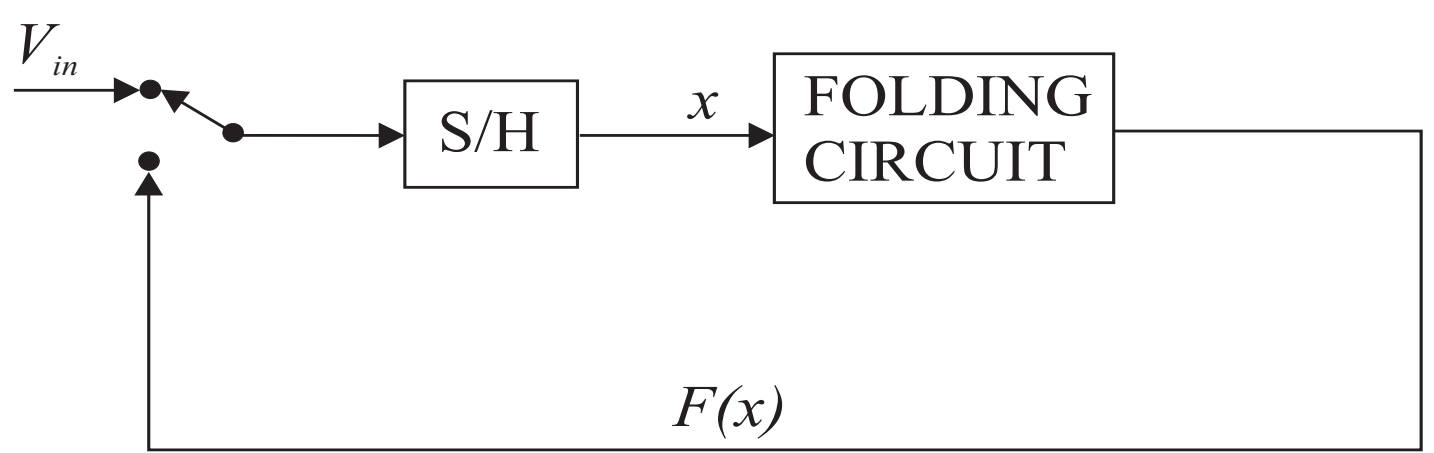

Fig. 1 

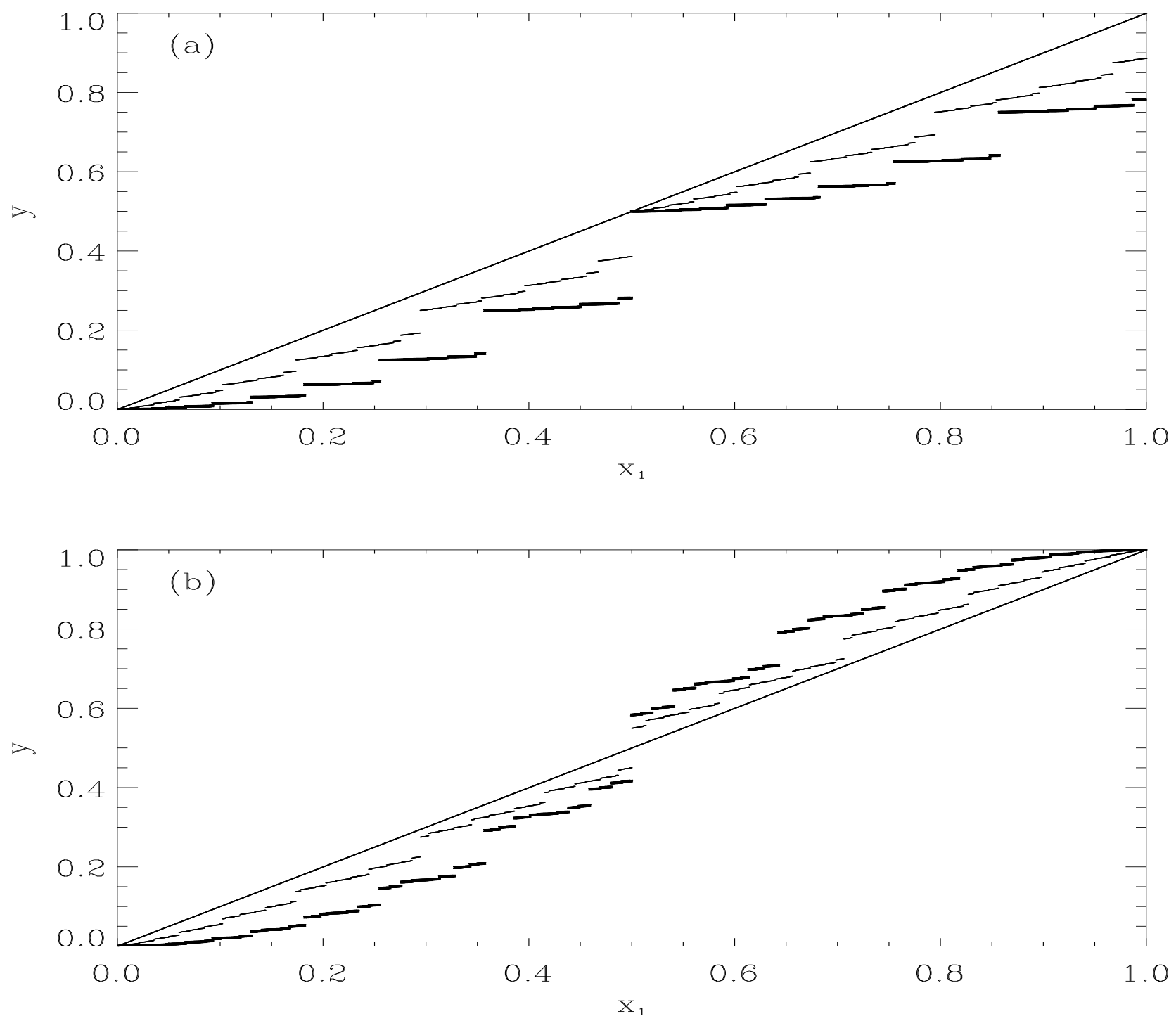

Fig. 2 

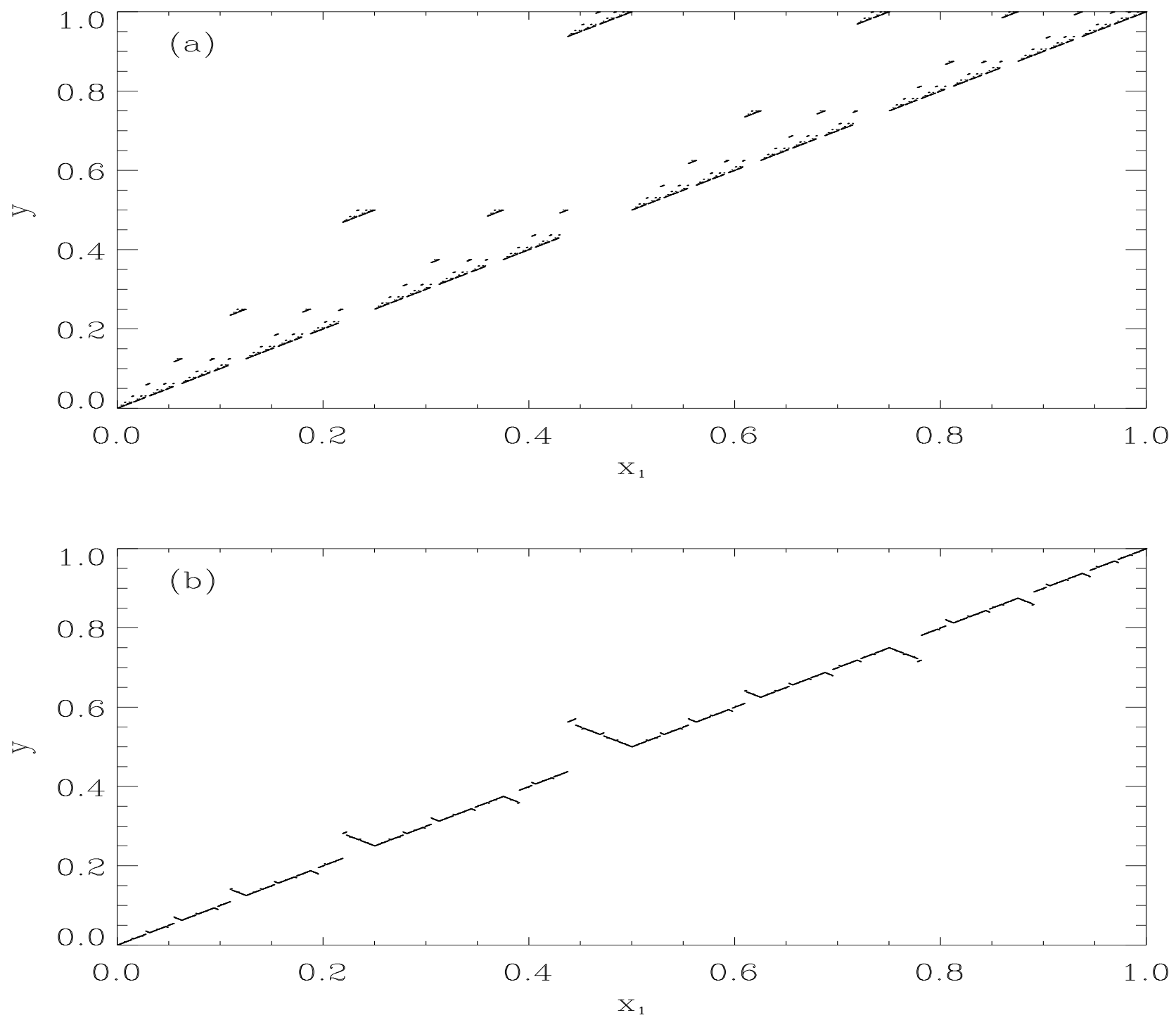

Fig. 3 


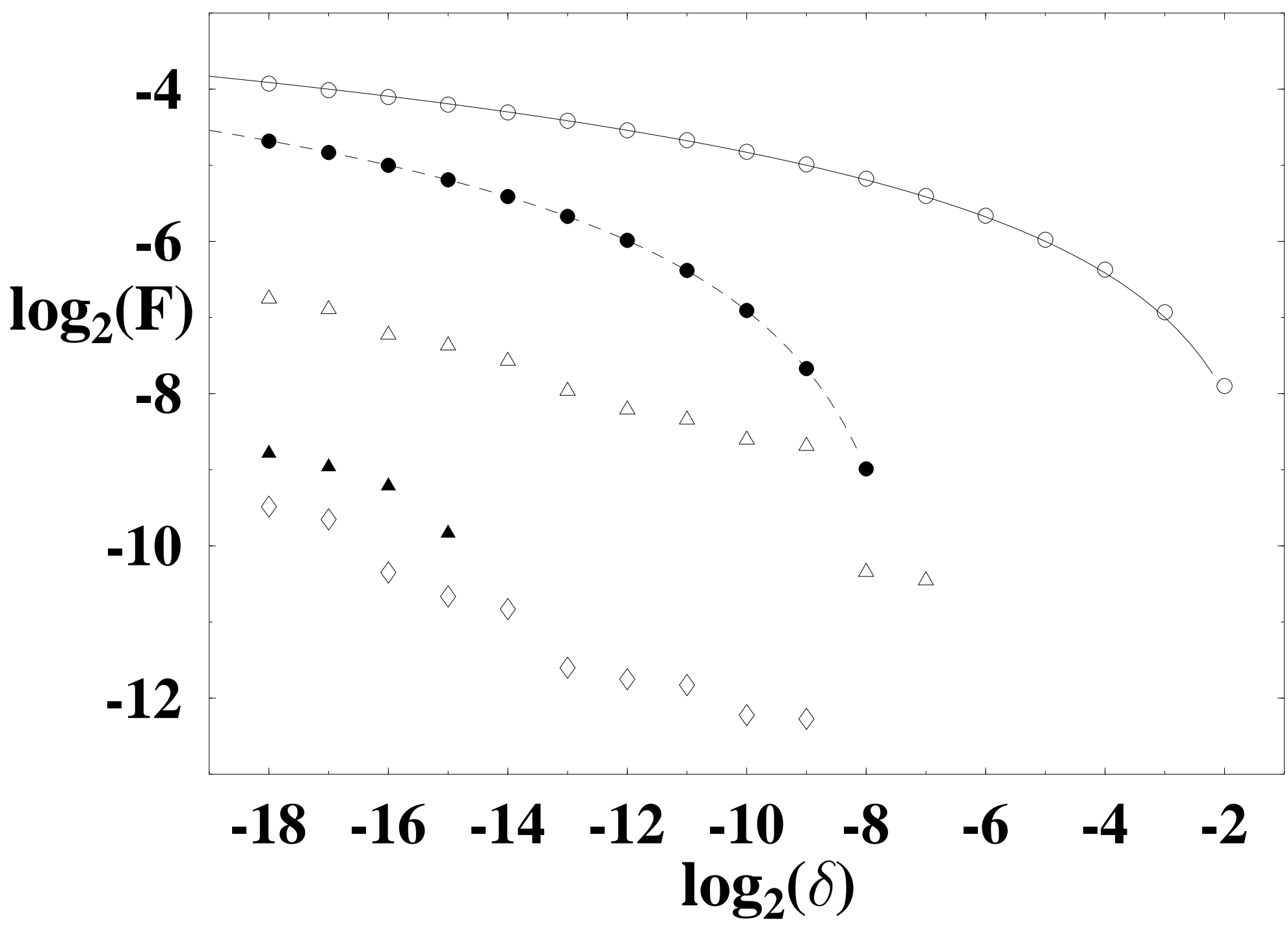

Fig. 4 